\title{
Explorando los efectos de la música del cine en la infancia
}

\section{Exploring the effects of film music in childhood}

\author{
Amparo Porta \\ Universidad Jaume I. Castellón. Departamento de Educación. \\ porta@edu.uji.es
}

Recibido: 17 de noviembre de 2012

Aprobado: 17 de junio de 2013

\begin{abstract}
Resumen
El escenario de la música ha sufrido importantes cambios en los últimos cincuenta años, teniendo en el desarrollo tecnológico y los mass media algunos de sus elementos determinantes. El artículo se interesa por el significado y el sentido de la música de cine en la infancia. Este medio sonoro requiere, por su carácter audiovisual y masivo, de herramientas que lo consideren como discurso, por ello se ha utilizado el análisis musical para saber qué se escucha, el sociológico para explicar cómo es y está construida su música y el semiótico para acceder a su sentido y significado, finalidad última del trabajo. El artículo termina con el análisis de la película Happy Feet, explorando la música en la historia, su relación con los dominios culturales, metáforas y asociaciones, relación con los valores y tendencias ideológicas. Este trabajo puede ayudar a reflexionar sobre algunas funciones sociales y educativas de la música en la infancia como forma de representación del mundo, aportar algunas razones sobre el valor de la expresión musical y artística en el currículo, proporcionar algunas herramientas para su estudio así como contribuir a la toma de conciencia en la producción cinematográfica y sus repercusiones.
\end{abstract}

Palabras clave: banda sonora, análisis, escucha, educación, cine infantil.

Porta, A. (2013): Explorando los efectos de la música del cine en la infancia. Arte, Individuo y Sociedad, 26 (1) 83-99

\begin{abstract}
The field of music has undergone a number of important changes in the last fifty years, mainly as a result of the developments made in technology and the mass media. The paper focuses on the sense and the meaning of film music in childhood. Due to its nature as a massive audiovisual sound medium, it requires tools that consider it as discourse. Thus, musical analysis was used to determine what is listened to, sociological analysis was taken to explain what it is like and how its music is constructed, and semiotics was applied to access its sense and meaning, which is the ultimate aim of the study. The paper ends with an analysis of the film Happy Feet, which sought to explore the music in the story, its relationship with the cultural domains, metaphors and associations, as well as its relation with values and ideological tendencies. This research can help gain new insights into some of the social and educational functions of music in childhood as a way of representing the world. Similarly, it can be useful in explaining the value of musical and artistic expression in school curricula, provide some instruments with which to study it, and also help raise awareness about film production and its repercussions.
\end{abstract}

Key words: soundtrack, analysis, listening, education, children's films. 
Porta, A. (2013): Exploring the effect of film music in childhood. Arte, Individuo y Sociedad, 26 (1) 83-99

Sumario: 1. El escenario de la escucha cotidiana, 2. Aproximación metodológica. Cómo saber qué escuchan los niños en la música de cine 3. Metodología. 4. Un estudio de caso. La música de la película Happy Feet. A modo de conclusión. Referencias

Este artículo recoge resultados de la investigación: 1) Proyecto «Estudio transversal de elementos de la programación televisiva infantil latinoamericana y su banda sonora por medio del análisis cualitativo y cuantitativo». P11B2010-37 financiada por I+D Local, 2010 a 2012; 2) Proyecto «La televisión como habitat sonoro. Estudio de los efectos de la banda sonora y sus narrativas audiovisuales en la infancia» Referencia: Edu2012-36404 financiada por I+D Nacional 2013 a 2015. Proyectos de Investigación, Subprograma de Proyectos De Investigación. Fundamental No Orientada. Convocatoria 2012.

\section{El escenario de la escucha cotidiana}

Una revisión del entorno sonoro actual indica un cambio sustancial en el hecho musical. La música que escuchan los niños en los mass-media es fruto de un proceso histórico determinado por cambios sociales, culturales, industriales y expresivos que se precipitan en el S. XX. Sobre ellos descansan una buena parte de los elementos del hábitat sonoro que han tenido como consecuencia la consolidación de los nuevos soportes comunicativos que vinculan la música con la imagen, la narración y el movimiento. En este complejo conglomerado destacan tres ejes que delimitan el escenario de la escucha cotidiana: la banda sonora, los contornos difuminados de las formas de expresión con su consecuente pérdida de la frontera, y su carácter mediático y espectacular. Este trabajo habla del primero de ellos pero requiere para su comprensión de los otros dos, porque juntos producen el sentido en el niño que acude al cine, ve la película, se sumerge en la historia y escucha su banda sonora. Nuestro tema se interesa por el significado y sentido de la música del cine como parte del entorno cotidiano del niño actual en la aldea global (Ramonet, 1998).

Las tendencias artísticas actuales distinguen entre música y arte sonoro. Para Reyes (2006) la diferencia principal entre ambas reside en que la música maneja un lenguaje en el que el sonido musical o tono está enmarcado dentro de escalas, contrapunto, armonía y ritmo, no así el arte sonoro que utiliza el sonido como elemento generador, lo libera y agrupa de nuevo. Por ello, la música no ha de depender de las tecnologías del audio, sin embargo el arte sonoro - dice- surge de la relación entre música, arte y tecnología. Reyes expresa esa interacción como dos triángulos unidos por los vértices. La intersección coloca música y sonido en una nueva interfaz de carácter expresivo o gestual donde los elementos sonoros tienen sentido en función del resto de sus elementos y, lejos de ser independientes, actúan como una suma de fuerzas, tal como se postuló desde las vanguardias artísticas.

Cuando aplicamos estos principios a la banda sonora, podemos observar cómo es el espacio de la escucha (Fig.1): 


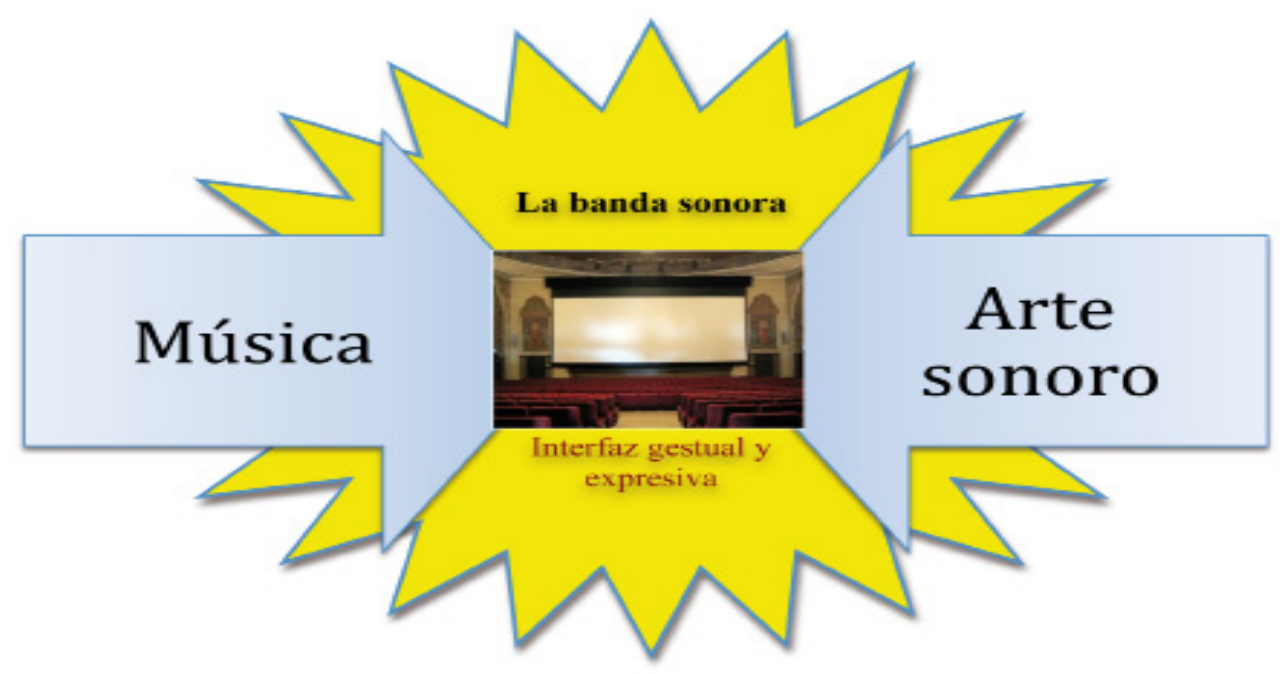

El espacio de la escucha

1. Medio de Comunicación de Masas

2. Creación industrial, política y/o institucional

3. Utiliza el lenguaje de la música adaptado al audiovisual

4. Está relacionada con la imagen y la narración, creada a partir de ellas

5. Incluye: artefactos sonoros, instalaciones, cración de ambientes, ruidos y aparatos

6. Sujeto a audiencias, publicidad, programación, asociada a productos y patrocinadores, elaboración de programación propia y estudios

7. Utiliza las estrategias de la comunicación y la publicidad

Figura 1. La banda sonora como espacio de la escucha

Este cambio de peso en las tendencias musicales y sus formas masivas de difusión se inició en el primer tercio del S. XX, supuso un cambio tanto en la producción como en la difusión musical equiparable a la aparición del Ars Nova en el S. XIII para Pérez (1981), siendo su detonante la aparición del rock and roll y los mass media (Porta, 2007). El segundo de los elementos es la pérdida de las fronteras o los contornos difuminados de las formas de expresión. Las tendencias musicales del S. XX incorporaron, entre otros elementos, el sonido no musical. Así, Boulez (1986), John Cage (1961) o Martenot (Nattiez, J, 1990), hablan del carácter esencial de éste en la obra, o de la música como contrapunto de sonido y silencio. Este planteamiento es el dominante hoy en día, especialmente en los espacios masivos de la difusión musical. Las tendencias contemporáneas del arte incluyen música, expresión verbal, instala- 
ciones, proyección sonora, artefactos, performance y expresión corporal, por citar algunos. Todo ello mediante soportes y medios que tienen en la electricidad, el agente último de la reproductibilidad técnica (Benjamin, 1989). Los cuatro componentes sónicos, música, sonido, silencio y escucha, han sido explicados desde la Teoría del Arte teniendo un apartado destacado en las vanguardias artísticas musicales y plásticas (Hauser, 1963; Benjamin, 1973; Adorno, 1972; Reyes, 2006), sin embargo en educación disponemos de pocos estudios sobre ello. Podemos decir que conceptos tan familiares cuando hablamos del sonido en términos de los mass media como: sonido grabado, bucles, simultaneidad y manipulación sonora, edición, cambios de velocidad, lenguaje fonético expresado por objetos, y otros, como estirar, comprimir, voltear, manipular, ver el sonido, percibir el ritmo visual, han formado parte del arte de las vanguardias del S. XX y también de los usos generalizados de la expresión juvenil como una tendencia hasta la actualidad. El tercer elemento destacado es su carácter mediático y espectacular, a veces convertido en un rito, como en el caso de los conciertos de rock, donde tienen lugar las grandes manifestaciones de la música popular urbana, estudiados por la Teoría de la Comunicación y la Etnomusicología (Lévi Strauss, 1969; Cruces, 1999). La música llega a todos por medio de formas de difusión masivas que utilizan estrategias publicitarias sometidas a controles de calidad industriales (Kingcheloe, 2000), son recibidas de forma anónima (Benjamin, 1989) y producen efectos poco estudiados en educación (Hearbreaves, 1999).

Estas líneas suponen un intento de comprensión del entorno sonoro del niño actual que tiene como elementos destacados la relación con la imagen, el carácter espectacular y su influencia social y educativa. El elemento central de nuestro estudio es la escucha de este entorno cotidiano y observamos falta de definición y de herramientas para su estudio.

\subsection{La banda sonora, como espacio de la escucha}

Diferentes corrientes y autores han establecido clasificaciones sobre las funciones de la banda sonora en el cine. Algunos distinguen entre los usos estéticos de la música según su conexión sentimental y localización espacio temporal (Cuellar, 1997), otros ponen el énfasis en su finalidad (Baetens, J., 2012: 406-407), en crear una atmósfera de época y lugar más convincente, o bien hablan de reforzar nuestras reacciones emocionales ante las escenas filmadas e implicar un elemento psicológico mucho mejor de lo que lo puede hacer el diálogo (Copland, 1952). Desde un ángulo de mayor permeabilidad social, Prendergast afirma que la música puede ser usada para subrayar o crear apoyaturas psicológicas siempre sutiles, porque la música tiene la habilidad catalítica de cambiar la percepción de la audiencia sobre las imágenes y las palabras, además de ayudar a construir un sentido de continuidad en el film, uniendo un medio visual que está, por su propia naturaleza, continuamente en peligro de desmoronarse (Prendergast, 1977). Podemos decir que la mayoría de los autores consultados coinciden en establecer que la problemática sobrepasa la música, y que su construcción y significado se produce por la suma de lenguajes (Porta, 2007). La banda sonora en el cine ha sido estudiada por diferentes autores como Michel Chion (1994) quién ha considerado la unidad entre la creación audiovisual y el sonido en la pantalla o Eisenstein 
para quien la banda sonora era algo crucial en la producción de cine porque en ella confluyen el texto oral, la música ambiental o incidental y los efectos sonoros. Los estudios comunicativos, discursivos y semióticos de la música han sido propuestos desde diferentes corrientes, entre las que destaca la Escuela de Frankfurt con autores como Adorno (1972), W. Benjamin (2003) o E. Gombrich (1979). En España encontramos los estudios realizados por J. Lozano (1982), González Requena (2006) o J. Talens y S. Zunzunegui (2007). En educación, cuando hablamos de la banda sonora y sus efectos en la infancia encontramos diferentes autores que lo han abordado de forma general desde varias ópticas, como Medrano (2008) en relación a los valores, y Herrera, Cremades \& Lorenzo (2010) que, con Novas, Iborra y Sampascual, (2007, 132) se han interesado por las preferencias musicales de los adolescentes. De forma específica desde la educación musical sobre las características de la banda sonora, encontramos los estudios de Porta $(1998,2007,2010)$.

\subsection{Presentación del problema}

Este trabajo forma parte de un estudio más amplio sobre el entorno musical cotidiano del niño, centrado hasta el momento en la televisión y el cine infantil. La finalidad es conocer los contenidos, influencia y efectos de la música que ofrecen los mas-media y sus repercusiones para la educación. La música cotidiana proporciona al niño elementos cognitivos, sociales, emocionales y patrimoniales a través de la oferta mediática (DeNora, T. 2000). Por ello es necesario conocerla para encontrar alternativas desde la educación y la producción (Del Río, Álvarez y Del Río, 2004). Sus repercusiones desde la educación musical son que producen efectos en el pensamiento sonoro y en las características de la escucha (Schafer, 1977; Schaeffer, 1966; Sloboda, 2005); desde las corrientes postestructuralistas hablan de efectos en la construcción de la conciencia (Vygotsky, 1981) y finalmente, desde la Teoría del Arte señalan su influencia sobre la representación del mundo (Gombrich, 1979). Este trabajo trata de conocer cómo es la música que escuchan los niños en el cine. Para ello requiere de una aproximación musical específica (Gómez-Ariza, 2000), que ayude a entender la escucha como construcción social (Vygotsky, L. S., 1981) y con sentido (Lozano, J., Abril, G., \& Peña-Marín, C., 1982). La aproximación musical es necesaria porque la música habla desde su propio lenguaje (Porta, 2007: 40). Conocer la música que ofrecen los mass-media supone analizar sus contenidos e intenciones como paso previo al objetivo final: conocer el sentido y significado para el niño que escucha la música del cine como parte de su proceso constante de evolución y aprendizaje. Por ello, este estudio tiene la mirada puesta en sus contenidos musicales, en la representación del mundo que propone y las repercusiones para el sujeto de la educación.

En la revisión de la literatura, desde el ámbito de las corrientes psicológicas destacan las aportaciones de la Psicología Cognitiva sobre el procesamiento de la información y las teorías de la reestructuración, ambas con una clara concepción «antiasociacionista», con autores como Piaget (1972), Vygotsky (1981) o la Escuela de la Gestalt (Peirce, C. S., 1956). La diferencia clave entre ellas radica en la unidad de análisis que utilizan, mientras la primera es elementarista, el enfoque cognitivo parte de unidades molares (Pozo, 1989:166 167). De ellas destacamos la Teoría socio histórica de Vygotsky, sometida a un proceso de reinterpretación y posible aplicación 
a la comprensión audiovisual según Korac (1988). El autor ruso muestra cómo los procesos mentales se explican por los instrumentos y signos que actúan de mediadores, poseedores de significado y, desde una perspectiva semiótica, habla de la internalización como un proceso de dominio sobre las distintas formas de signos externos (códigos). Para diferentes autores la noción de mediación es la contribución más original e importante de Vygotsky. La argumentación principal sobre los elementos mediacionales se apoya en el estudio sobre la naturaleza comunicativa de los signos. El término signo es un concepto utilizado por el autor con el sentido de "poseedor de significado". Es necesario que todo aquello que es interno en las formas superiores haya sido externo, es decir, que fuera para otros lo que ahora es para uno mismo. Toda función psicológica superior atraviesa necesariamente una etapa externa en su desarrollo, ya que inicialmente es una función social. (Vygotsky, 1981:162). De igual modo, desde la Pedagogía Crítica, Giroux dice que el lenguaje es el lugar donde las formas reales y posibles de organización social así como sus posibles consecuencias se definen controvertidas. Sin embargo, es también el lugar donde se construye el sentido de nosotros mismos, nuestra subjetividad (Giroux, 1991: 76). Por ello hace una recomendación educativa: ser culturalmente alfabetizado es tener la información básica necesaria para prosperar en el mundo moderno (Giroux, 1991: 43). McLaren, P., \& Kincheloe, J. L., (2008) manifiestan que los cambios experimentados durante la infancia en las sociedades occidentales contemporáneas plantean nuevos retos. La proliferación de tecnologías, la privatización de los medios y el espacio público, así como la polarización entre los ricos y los pobres cibernéticos hacen necesaria una nueva estrategia para proteger los derechos de los niños como ciudadanos y consumidores. Los aspectos sociales que definen al "otro" desde una perspectiva pedagógica multiculturalista, buscan entender los mecanismos de opresión y discriminación, o de libertad y reconocimiento de las minorías raciales, de clase social y de género que se reproducen a través de la construcción y transmisión de conocimientos, valores e identidades en ámbitos sociales distintos. La pedagogía crítica -dicen- debería responder a un sistema educativo en permanente estado de confusión, donde las políticas sociales y educativas sólo interesan en términos de cómo adaptarlas a las necesidades del mercado. En esta línea encontramos algunas aproximaciones metodológicas, como la de Franz, T.S. (2002). Sobre las funciones psicológicas que cubre la música en la vida cotidiana Hargreaves (1999) dice que la respuesta a esta pregunta está cambiando como resultado de los actuales cambios sociales y tecnológicos en la música misma, y cómo estos cambios nos obligan a re-evaluar el papel de su contexto social en términos de los dominios cognitivos, emocionales y sociales. Para el autor los resultados de sus investigaciones les llevan a concluir que la función social de la música se manifiesta en tres formas principales para el individuo: la gestión de la propia identidad, las relaciones interpersonales y el estado de ánimo. Por ello propone una nueva agenda para la psicología de la música que ponga la dimensión social en el centro, y considere el contexto interdisciplinar, los efectos de la "democratización" de la música, el papel de la teoría, la relación entre teoría y práctica, y las implicaciones para la metodología de la investigación. 


\subsection{Qué escuchan los niños en el cine infantil}

La pregunta de investigación es ¿Qué escuchan los niños en el cine infantil? La intención es identificar el entorno sonoro cotidiano para conocer su influencia, efectos y busqueda de alternativas. Las áreas de conocimiento implicadas son: la Historia y Teoría de la Música, la Comunicación, la Semiótica, la Sociología, el Arte y la Educación. Los textos y discursos analizados en el proyecto son la música en publicidad, la banda sonora del cine y la programación infantil de televisión (Porta, 2007, 2010, 2011). Este artículo se centra en el cine infantil.

\section{Aproximación metodológica. Cómo saber qué escuchan los niños en la música de cine}

\subsection{El medio sonoro}

Desde diferentes aproximaciones se han desarrollado vías para conocer cómo se produce la escucha de la música y sus efectos. El entorno sonoro tiene unas características propias de nuestro momento histórico: urbano, audiovisual, tecnológico, industrial, globalizado y de consumo. Revisando la bibliografía se observa que la problemática que abordamos no es nueva. Las aproximaciones a la escucha ha sido una preocupación para todos aquellos que trabajan con el sonido, ya sean músicos, físicos o arquitectos. Estos últimos han puesto su atención en diferentes ocasiones sobre el entorno sonoro, otorgándole una importancia social pertinente en nuestra exploración. En sus trabajos indican que cada fragmento del tejido urbano posee unos rasgos sonoros característicos que nos hablan de sus cualidades espaciales, de las temporalidades y de los usos que lo habitan, y estos rasgos constituyen su identidad ordinaria, cotidiana. Sobre la escucha musical, Atienza (2004:161) habla de que necesitamos recurrir a una nueva escucha musical del entorno cotidiano, para lo cual hemos de apoyarnos en el lenguaje y en los instrumentos de análisis que la música ha desarrollado. Las nuevas necesidades del contexto urbano necesitan soluciones específicas adecuadas al entorno actual, cuyos antecedentes los encontramos en diferentes esfuerzos interdisciplinares de los últimos 50 años. Durante las décadas de 1960 y 1970 aparecen dos acercamientos. El objeto sonoro de Pierre Schaeffer, parte de un análisis fenomenológico del universo audible centrado sobre los modos de percepción auditiva (Schaeffer, 1966), y la noción de paisaje sonoro de Robert Murray Schafer como representación del entorno sonoro que podríamos calificar de «compositiva». Estos autores se preguntan por nuevas soluciones para nuevas necesidades que, dicen, necesitan una revolución entre los campos de los estudios sonoros. Para Schafer la revolución consistirá en la unificación de aquellas disciplinas que se ocupan de la ciencia del sonido y aquellas que se ocupan del arte del sonido. El resultado será el desarrollo de las interdisciplinas ecología acústica y diseño acústico (Schafer, 1977). Desde perspectivas más actuales, se ha ido ampliando de manera progresiva el campo y así, Atienza (2004) indica que el problema reside en la definición del objeto sonoro que debe constituir la unidad elemental de un «solfeo sonoro», pero carece de una escala adecuada para el análisis de configuraciones tan complejas como las 
urbanas. En la misma línea, Augoyard y Torgue (1995: 6-7) hablan del concepto de paisaje sonoro y incide en que presenta ciertas limitaciones para el análisis del contexto urbano, que tiene su origen en una escala de trabajo excesivamente extensa.

Desde una posición educativa, y de acuerdo con Atienza (2004), el objeto sonoro no es un objeto en sí, ni se ocupa de la materia sonora en cuanto objeto, sino en cuanto medio. Persiste pues la interrogación acerca del propio sonido, abordada por Pierre Schaeffer y Robert Murray Schafer. No se trata de estudiar el sonido como finalidad en sí mismo, desvinculado de todo contexto; al contrario, el estudio se realiza en función de un tiempo y espacio precisos. Es, precisamente, Jean-François Augoyard (1989: 21) y el laboratorio Cresson, quienes se presentan como herederos y complementarios de las dos anteriores. Esta línea investigadora surge de la necesidad de encontrar una herramienta interdisciplinar adecuada a la escala de una configuración urbana que permita integrar otras dimensiones que la puramente estética (Atienza, 2004). El laboratorio Cresson de Grenoble establece un protocolo de encuesta que indica un cambio de paradigma que reposa sobre tres tipos consignas básicas: consignas relativas a la descripción, al recorrido y a la experiencia. La aportación principal para nosotros es que los parámetros han sido ampliados e incluyen los datos contextuales y también los más propios, los experienciales. Así, pues, no hablamos sólo de música o arte sonoro, añadimos medio sonoro y además "situado". Y, en esta nueva definición del espacio de la escucha se ven afectados los contenidos de la proximidad (De Moragas, 2007). Y también el valor comunicativo, estético y patrimonial de la música en la educación (BOE, 2006 y 2007).

Este es el marco general del objeto de estudio, su contenido destacado es la escucha del hábitat sonoro contemporáneo del que se ha tomado una parte, el cine infantil, de procedencia industrial, alto impacto y refuerzo que utiliza fórmulas musicales, sonoras y comunicativas adaptadas al medio audiovisual. Como resumen del apartado podemos decir que el medio sonoro dispone de música, está creado por principios de diseño industrial urbano con antecedentes en la Bahaus (Westerkamp, H., 1994). Está sujeto a los intereses de los mass-media, forma parte del contexto, es decir se produce por inmersión, necesita de herramientas multidisciplinares para su comprensión y alternativas que incluyan elementos descriptivos, contextuales y experienciales.

\subsection{Recorridos, secuencias y líneas de tiempo}

Pero la investigación empírica requiere del uso de instrumentos. La música se produce en el tiempo, por ello la exploración ha de tener un anclaje temporal que ha de ser doble para responder a qué se escucha y dónde está situado aquello que se escucha. En este caso, la primera cuestión a abordar son sus acotaciones para ser observada y analizada de forma objetiva. El protocolo de encuesta del laboratorio Cresson, desde la arquitectura nos orienta:

- La descripción: qué se escucha

- El recorrido: dónde está situado

- La experiencia: desde dónde se escucha (Porta, 2007) 


\section{Metodología}

Como metodología de análisis, hemos utilizado el análisis musical (Zamacois, 1987), el sociológico (Ibañez, 1979; Kripendorff, 1990) y el semiótico (Talens, 1995). (Fig.2)

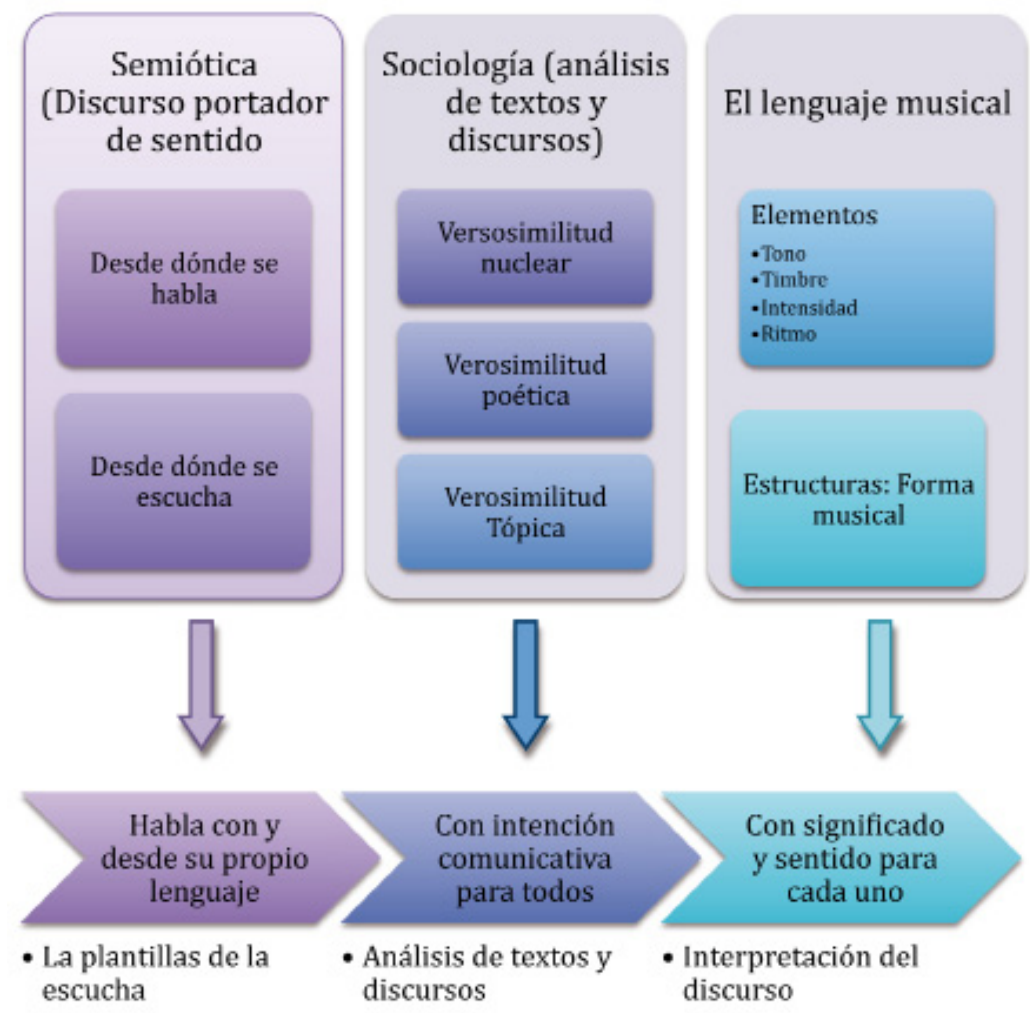

Figura 2. Aproximación metodológica. Porta, A. (2007) Músicas públicas, escuchas privadas. Hacia una lectura de la música popular contemporánea p.73

La Música habla desde su propio lenguaje. Por ello hemos estudiado los elementos presentes en el entorno sonoro -tono, timbre, intensidad y ritmo-, su estructura -la forma musical- y opciones estilísticas y tímbricas, (Zamacois, 1987).
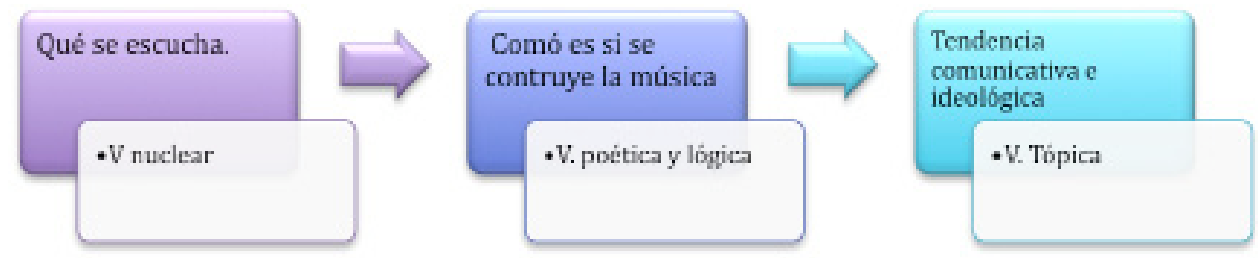
Figura 3.- La música habla para todos. Niveles de verosimilitud

La Música habla para todos (Fig.3). Mediante el análisis sociológico de textos y discursos hemos accedido a aquellos elementos que actuan como síntomas de aspectos no observables desde el análisis musical. El discurso sustituye la verdad, inalcanzable, por la verosimilitud. De igual modo, en música, el mundo es sustituido por su discurso al igual que las cosas son sustituidas por las cualidades sonoras. Por ello nos hemos acercado desde tres grados de aproximación: la verosimilitud nuclear, la verosimilitud poética y lógica, y la verosimilitud tópica (Kripendorff, 1990). La verosimilitud nuclear nos muestra la parte más interior del texto musical, por medio de categorías hemos contestado a qué se escucha. Posteriormente, la verosimilitud poética y lógica nos ha mostrado la organización en la cadena comunicativa; en nuestro caso mediante la forma musical, los géneros musicales y algunos rasgos estilísticos y tímbricos. Desde este segundo nivel, hemos respondido a dónde está situada y cómo se construye la música. Mediante el tercer nivel, la verosimilitud tópica, hemos visto la tendencia comunicativa del discurso en su conjunto, su posición ideológica.

La música es recibida de forma individual y privada. El análisis semiótico ha permitido acceder a la escucha, reducto, como decíamos, privado y frágil, al cual, de forma paradójica, va dirigido todo el aparato tecnológico e industrial de la Posmodernidad, en este caso, el niño como espectador de la película, y opera desde dos lugares: el lugar desde dónde habla y el lugar desde dónde se escucha (Porta, 2007). Este último es el elemento más anónimo y a la vez más determinante del fenómeno que analizamos.

El procedimiento general ha consistido en aplicar los patrones establecidos por la Teoría de la Música a diferentes productos audiovisuales dirigidos a la infancia. Los resultados han constituido la base del análisis de contenido para llegar finalmente a la interpretacion semiótica del discurso resultante. A través de todo ello nos hemos acercado a las características discursivas del cine infantil, sus peculiaridades, contradicciones y tendencias.

\section{1 El método y la codificación}

Este trabajo forma parte de una investigación más amplia que tiene como objetivo conocer qué escuchan los niños en el entorno cotidiano. En este caso nos ocupamos de conocer la banda sonora del cine y sus efectos en la infancia. El marco teórico de referencia para su interpretación es el semiótico porque el estudio se interesa por el sentido, es decir por la posición del sujeto en el discurso (Lozano, 1982; Talens, 1995; Zunzunegui, 1989). Para la fundamentación metodológica en semiótica musical nos hemos apoyado en Adorno (1972); Molino, 1975 y Nattiez, 1990; Gauthier, J, 1996). Finalmente, para el análisis, hemos seguido el procedimiento descrito por Umberto Eco. El autor dice en su introducción a La Estructura Ausente (Eco y Cantarell, 1978) que una investigación semiótica solamente tiene sentido si la estructura del campo es asumida como una entidad imprecisa que el método se propone aclarar. Por ello propone como metodología: 1) Cotejar definiciones, 2) Establecer el campo semiótico en términos de vastedad y desorden, y 3) Crear un modelo de investigación sometido a contradicción. De esta forma, si la operación tuviera éxito, se lograría algo que casi suena utópico "mantener la complejidad del campo confiriéndole una 
estructura, y por lo tanto, transformar el campo en un sistema. Así, pues, siguiendo a Eco, nuestra hipótesis operativa es transformar el campo, la banda sonora del cine infantil, en un sistema.

\section{2 El modelo}

Como modelo específico, hemos utilizado el propuesto por Gómez-Ariza (2000). Los modelos que intentan explicar la manera en que la música está representada mentalmente, varían en el rango de fenómenos que explican y en las unidades de representación que postulan (Gómez Ariza, 2000). Uno de los primeros problemas para juzgar estas teorías o proponer otras nuevas, es delimitar cuáles son las características de la música que determinan la manera en la que percibimos y, posteriormente, la recordamos. Sin embargo, hay pocos trabajos que, de forma sistemática, revisen cada uno de los factores que componen la música y la manera en que influyen en la percepción y el recuerdo. Una de las dificultades según Gómez-Ariza, es que no existe un marco teórico unificador que permita organizar los datos existentes. Para conocer la banda sonora del cine infantil y sus efectos en la infancia nos hemos apoyado en estos autores que consideran los estímulos musicales compuestos de un gran número de dimensiones que se combinan para formar el patrón auditivo complejo que forma la música. Utilizando este modelo hemos estudiado algunos largometrajes de cine infantil, de forma individualizada como análisis de caso.

\section{3 Los instrumentos}

Para contestar a qué se escucha en la música de cine, cómo es, se construye y qué efectos produce, se han utilizado tres instrumentos: la selección de secuencias, el análisis musical y las categorías. Los dos primeros nos sirven para ubicar las obras musicales en el film, para ello hemos establecido el cronograma, situado sus elementos y la música. La palabra situado, de nuevo aparece como un concepto clave para la comprensión narrativa, la música en la historia, ya que ha de estar anclada para ser interpretada. El tercero han sido los indicadores musicales.

Se han considerado 13 indicadores correspondientes a las cualidades sonoras más representativas susceptibles de medición, ya validadas y aplicadas a distintos documentos audiovisuales. Se trata de las siguientes variables de análisis: Sin sonido, sonido no música, sonido musical, voz, métrica y rítmica, tipo de comienzo, dinámica, agógica, género y estilo, organización sonora, cadencia, modulación, textura sonora, plano sonoro, jerarquías tonales (Porta, Ferrández, 2009), y como unidades de análisis se han considerado los números musicales y las canciones de la película.

En esta primera parte hemos realizado una aproximación a la banda sonora del cine infantil desde una mirada comunicativa. Nuestro interés se centra en el lado de la escucha, por ello hemos delimitado nuestro campo de acción y pregunta de investigación ¿Qué escuchan los niños en el cine infantil? Para contestarla hemos seleccionado el paradigma, el modelo, la metodología y situado sus elementos. Por medio de los niveles de verosimilitud del discurso hemos podido contestar a nuestras preguntas ¿Qué se escucha?¿Cómo es y se ha construido su música, y dónde está situada? 
Finalmente, como consecuencia de todo ello, hemos entendido un poco mejor cuál es la representación del mundo que ofrecen algunas bandas sonoras del cine infantil e indagado sobre sus posibles efectos.

\section{Un estudio de caso. La música de la película Happy Feet}

La música del cine requiere de estrategias específicas para su comprensión. El cine infantil muestra a lo largo de sus noventa minutos una selección de música con la que crea una parte importante de la continuidad y sentido de la acción narrativa, sus vinculaciones emocionales, de valores y opciones culturales. Las películas constituyen discursos audiovisuales que han de ser considerados como estudios de caso para poder responder a qué música se escucha y cómo es. Los tres niveles, descriptivo, comunicativo y de sentido para el espectador, explicados en la Figura 1, nos ha proporcionado información contextualizada del discurso sonoro. En esta segunda parte hemos aplicado los principios y metodología a la película Happy Feet. Mediante la verosimilitud nuclear hemos estudiado las características de la música de su banda sonora, la verosimilitud poética y lógica hemos conocido como está construido el discurso musical, y través de todo ello hemos accedido a la verosimilitud tópica, es decir, sus tendencias discursivas e ideológicas (Porta, 2007).

\subsection{Muestra, procedimiento y diseño de instrumentos}

Happy Feet es una película de animación estrenada a finales de 2006, dirigida por George Miller, producida por Warner Bros, ganadora de un Oscar y un Globo de Oro e incluye las voces de Elijah Wood, Robin Williams, Brittany Murphy, Hugo Weaving, Nicole Kidman y Hugh Jackman. Para su estudio se realizó un visionado completo de la película, se ubicaron sus números musicales en el eje cronológico y la acción narrativa con sus personajes, espacios escenográficos y grupos culturales. En el análisis se aplicaron los niveles de verosimilitud y se realizó un estudio de las categorías musicales para conocer, como indicadores, sus opciones rítmicas, tímbricas, de textura, etc. En este nivel de concreción se aplicó la plantilla de la escucha (Porta, Ferrández, 2009). Nivel 1. V. nuclear. Posteriormente, para acceder a la cadena comunicativa: Nivel 2, V. poética y lógica, se situaron los segmentos musicales, crono, personajes, géneros y estilos musicales. Se analizaron todos los números musicales y, de forma detallada, se estudiaron ocho canciones y tres números con rítmica coreografiada. Para ello se elaboró un cronograma descriptivo con las cuatro secciones de la trama narrativa, colocando las canciones en sus secciones y secuencia temporal. En último lugar, para conocer la presencia e incidencia comunicativa de la música en la historia, se estudió su presencia en la acción, tanto diegética (interpretada por los personajes) como no diegética (música en off) (Zunzunegui, 1989). Finalmente se realizó un análisis de los datos obtenidos, su interpretación y estudio de tendencias: Nivel 3. V. Tópica. 


\subsection{La banda sonora de Happy Feet y sus dominios culturales}

- La historia

La película muestra la vida en una sociedad de pingüinos que el film muestra por medio de tres grupos diferenciados: los pingüinos emperador, los pingüinos latinos y los humanos. Los primeros son los protagonistas de la película. La red de conflictos comienza con el nacimiento de uno de ellos porque nace diferente. Por esa razón se marcha de su sociedad de origen donde cantar es la acción más valorada y el no sabe, En el autodestierro, se encuentra con un grupo de pingüinos latinos que aprecian su mejor habilidad, saber bailar. La presencia de los tres grupos culturales (pingüinos emperador, pingüinos latinos y hombres) es desigual en la película. La presentación de los pingüinos emperador e inicio de la red de conflictos en su sociedad ocupa los 30' iniciales. La vida de los pingüinos latinos en su medio ambiente natural está desdibujada y es más breve (16'). En esta sección se muestra una sociedad que se divierte y resuelve sus problemas improvisando, con escasa capacidad de decisión y trabajo cooperativo en grupo, recurriendo finalmente a resolver los conflictos por medio de consultas al sabio. En cuanto al tercer grupo, los humanos, causantes del deterioro social y la alteración del ecosistema, aparecen en la última parte de la película, ocupando los últimos 3', en los que todo se resuelve con un final feliz.

- La banda sonora en la historia. Estudio de la música.

La película dedica 5 canciones a la Sección 1, ninguna a la Sección 2, 2 a la Sección 3 y 1 a la Sección 4. Son las siguientes:

\begin{tabular}{|c|c|c|c|c|c|c|c|c|c|c|}
\hline \multicolumn{6}{|c|}{ Sección 1} & \multicolumn{3}{|c|}{ Sección 2} & \begin{tabular}{|c|} 
Sec- \\
ción 3
\end{tabular} & $\begin{array}{c}\text { Sección } \\
4\end{array}$ \\
\hline \multicolumn{6}{|c|}{ Sociedad de pingüinos emperador } & \multicolumn{3}{|c|}{$\begin{array}{c}\text { Destierro y aparición } \\
\text { de pingüinos latinos } \\
47: 15\end{array}$} & \begin{tabular}{|c|} 
El en- \\
cuentro \\
$1: 30$
\end{tabular} \mid & $\begin{array}{l}\text { El hom- } \\
\text { bre } \\
1: 31: 58 \\
1: 34: 44\end{array}$ \\
\hline $\mathrm{C} 1$ & $\mathrm{C} 2$ & $\mathrm{C} 3$ & $\mathrm{C} 4$ & no canción & $\mathrm{C} 5$ & \multicolumn{3}{|c|}{ No canción } & $\begin{array}{lll}\mathrm{C} 6 & \mathrm{C} 7 \\
\end{array}$ & $\mathrm{C} 8$ \\
\hline $\begin{array}{l}\text { Elvis } \\
\text { Presley }\end{array}$ & $\begin{array}{c}\text { Coro } \\
\text { voces } \\
\text { blancas. }\end{array}$ & $\begin{array}{c}\text { Beach } \\
\text { Boys }\end{array}$ & $\begin{array}{l}\text { Some- } \\
\text { body }\end{array}$ & $\begin{array}{c}\text { Coros y } \\
\text { orquesta. } \\
\text { No meló- } \\
\text { dico. }\end{array}$ & $\begin{array}{c}\text { Coros y } \\
\text { Orquesta. } \\
\text { Coreografía } \\
\text { en el mar. }\end{array}$ & $\begin{array}{l}\text { Sólo ritmo: } \\
\text { Batucada, } \\
\text { recitado: } \\
\text { Mambo, } \\
\text { Rap }\end{array}$ & $\begin{array}{c}\text { Suenan } \\
\text { trompeta. } \\
\text { Aire } \\
\text { mejicano }\end{array}$ & $\begin{array}{c}\text { Coros } \\
\text { y or- } \\
\text { questa, } \\
\text { no me- } \\
\text { lódico }\end{array}$ & A mi & \\
\hline \multicolumn{6}{|c|}{ No diegético } & Diegético & \multicolumn{2}{|c|}{$\begin{array}{l}\text { No diegé- } \\
\text { tico }\end{array}$} & $\begin{array}{c}\text { Diegé- } \\
\text { tico }\end{array}$ & $\begin{array}{c}\text { No die- } \\
\text { gético }\end{array}$ \\
\hline
\end{tabular}

El dominio cultural de los pingüinos emperador prevalece durante toda la película mostrando una sociedad vertebrada y organizada. Pero lo más importante es que el protagonista nace en ella, se siente diferente y se marcha, pero quiere volver a casa. La película tiene cuatro secciones narrativas: 1) Presentación de la sociedad, 2) Destierro y encuentro con los pingüinos latinos, 3) Regreso a casa y 4) Aparición 
del hombre. Las tres primeras reproducen la estructura narrativa de la película "El Rey León" aunque no tiene las habilidades persuasivas/proyectivas de esta (Porta, 1998). La música crea un aura conservador, refleja el bien establecido de la sociedad de origen (pingüinos emperador) que es la referencia en la película. Desde el punto de vista estilístico utiliza canciones del pop/rock y algunas clásicas con polifonía de voces blancas en su selección de música diegética (interpretada por los personajes). En el reino de los pingüinos latinos no se escucha ninguna canción, sólo ritmos latinos con percusión (batucada y mambo). Su apuesta musical es más breve y débil por variedad, calidad interpretativa y asociación a personajes. La música no diegética de la película (la no interpretada por los personajes) simboliza la presencia del narrador en la historia, es acústica de orquesta, con predominancia de instrumentos de cuerda y presencia de trompetas en los momentos álgidos de la trama. En líneas generales es música incidental, descriptiva de la acción y tiene carácter poco melódico, salvo algunas secuencias con aires latinos. Destaca en este apartado una gran coreografía en el mar, uno de los mejores números de la película, con música de orquesta, no diegética y protagonizada por los pingüinos emperador. La música está formada por canciones conocidas, de éxito y se escucha con una calidad interpretativa e instrumental poco habitual en el cine infantil quedando siempre asociada al primer grupo étnico, excepto en "A mi manera". La banda sonora de la película resume la riqueza cultural de la sociedad que la película presenta como dominante y conservadora, atribuyendo al otro grupo, el de los pingüinos latinos, el valor de lo exótico y cómico.

Qué se escucha, cómo es su música y cuales son sus dominios culturales.

Para contestar estas preguntas se han utilizado los niveles de verosimilitud:

Verosimilitud nuclear. Elementos musicales utilizados en la película. La película utiliza obras tanto vocales como instrumentales con solistas, coros, instrumentos eléctricos originales del entorno del pop/rock y acústicos, tanto de orquesta (todas las familias) como populares y percusión corporal. Predomina la acentuación binaria $\mathrm{y}$ tiene intensidad fuerte en todas las escenas.

Verosimilitud poética y lógica. Ordenación de elementos musicales en la estructura narrativa. Las canciones tienen duraciones variadas y no se escuchan enteras, la organización musical se obtiene por fusión de varias melodías (dos o tres habitualmente) y son utilizadas tanto como figura como fondo. Los géneros y estilos que se escuchan son pop/rock inglés, americano y australiano, clásico y cinematográfico descriptivo. La película presenta una buena sincronía entre música e imagen, y todas las canciones son interpretadas por los propios personajes aunque sin presencia de la parte instrumental. Las voces en las canciones son muy distintas de la voz hablada de los personajes, lo que resta credibilidad a las escenas, los instrumentos que las acompañan quedan en plano no diegético, es decir, sin presencia en la acción.

Verosimilitud Tópica. Valoración de la banda sonora de la película, estudio de tendencias y dominios culturales. La banda sonora de la Sección 1, hace referencia al lenguaje musical como valor de una sociedad culta y desarrollada en la que tiene un lugar destacado el valor educativo y cultural de la música: cómo crear una canción, la escuela como lugar de aprendizaje musical o la música como patrimonio cultural, de cohesión social y el bien común. Sin embargo, en el reino de los pingüinos latinos la 
música se ofrece como diversión y evasión de los problemas de una sociedad falta de recursos, poco vertebrada y expuesta a los ataques de los depredadores.

Como resumen podemos decir que la música ha representado los valores de una sociedad dominante construida a si misma, entre todos, cuidando del bien común y ha asumido como propios una gran variedad de estilos y tímbricas de carácter universal, así como canciones reconocidas de éxito y algunas clásicas. Todas ellas se han identificado con el grupo de los pingüinos emperador que se presentan en la sección 1, siendo toda la música de la sección 2 (destierro) una aportación cultural que fortalecía al grupo dominante en la sección 3 (encuentro) y que muestra su triunfo en la sección 4 (aparición del hombre y defensa del ecosistema). La música, como metáfora de la sociedad, ha mostrado en la película un grupo dominante, el de los pingüinos emperador, que se identificaban con la cultura pop, incluían pinceladas de música coral clásica e instrumental cinematográfica descriptiva, y han absorbido una parte de la cultura extranjera para fortalecer su sociedad y favorecer su propio desarrollo económico y cultural.

\section{A modo de conclusión}

Este trabajo puede ayudar a reflexionar sobre algunas funciones sociales y educativas de la música en la infancia como forma de representación del mundo, aportar algunas razones sobre el valor de la expresión musical y artística en el currículo, y también contribuir a la toma de conciencia de las producciones cinematográficas y sus repercusiones. De igual modo proporciona algunas herramientas para su estudio.

\section{Referencias}

A Adorno, TW. (1972). Réflexions en vue d'une sociologie de la musique. Musique en Jeu, 7. Paris. Seuil.

Atienza, R. (2004). L'identité sonore: une variable essentielle dans la configuration urbaine. Grenoble, Cresson.

Augoyard J.F. (1989). Contribution à une théorie générale de l'expérience sonore: Le concept d'effet sonore, Revue de Musicothérapie, vol. IX, n. ${ }^{\circ}$ 3. París, Ed. Association française de musicothérapie.

Augoyard, J.F. y Torgue, H. (Eds.) (1995). A l'écoute de l'environnement. Répertoire des effets sonores. 174, 6-7. Marsella. Parenthèses.

Baetens, J. (2012). Beyond the Soundtrack: Representing Music in Cinema (review). Leonardo 41.4: 406-407. Project MUSE. Web. 9 Nov. 2012. <http://muse.jhu. $\mathrm{edu} / \mathrm{s}$.

Benjamin, W. (1936). Das Kunstwerk im Zeitalter seiner technischen Reproduzierbarkeit, en Zeitschrift für Sozialforschung, [Walter Benjamin, La obra de arte en la época de su reproducibilidad técnica, Discursos Interrumpidos. Buenos Aires. Taurus, 1989]

Boulez, P. (1986). Orientations, trad. M. Cooper, J.J. Nattiez (ed.). Cambridge: Harvard University.

Cage, J. (1961). Silence. Middletown: Wesleyan University. 
Chion, M. (1994). Audio-vision: sound on screen. Nova Iorque: Columbia University. Copland, A. (1952). Music and Imagination. Cambridge, Mass. Harward University. Cruces, F. (1999). Con mucha marcha: el concierto pop-rock como contexto de participación. Revista transcultural de Música, $n^{\circ}$ 4. http://www.sibetrans.com/trans/ trans4/cruces.htm

Cuellar, C. (1998). Cine y música: el arte al servicio del arte. Valencia. UPV,

De Moragas, M. (1991). Teorías de la comunicación, $5^{\text {a }}$ ed., Barcelona, Gilli.

Del Río, P. Alvarez, A. y Del Río, M. (2004). Pigmalión. Informe sobre el impacto de la televisión en la infancia. Madrid. Fundación Infancia Aprendizaje.

DeNora, T. (2000). Music in everyday life. Cambridge Univ Pr.

Eco, U. y Cantarell, F. (1978). La estructura ausente: Introducción a la semiótica, Madrid, Lumen.

Franz, T.S. (2002). Educación para la comprensión crítica del arte. un modelo de análisis. Arte, Individuo y Sociedad, 14, 27-47.

Gauthier, J. (1996). Veinte lecciones sobre el sentido y la imagen, Cátedra. Madrid.

Giroux, H.A. (1991). Postmodern education: Politics, culture, and social criticism Y1.

Gombrich , E.H. (1979). Arte e ilusión. Barcelona, Gustavo Gili, .

Gómez-Ariza, C. Bajo, T. y Puerta, C. (2000). Determinants of musical representation: Cognitiva, 12, 1, 89-110(22), Fundación Infancia y Aprendizaje.

González Requena, J. (2006). Clásico, manierista, postclásico. los modos del relato en el cine de hollywood. Comunicacion y Hombre, (2), 151-153.

Hauser, A. (1963): Historia social de la literatura y el arte. (Tr. A. Tovar y F.Varas). Madrid: Guadarrama.

Hargreaves, D.J., y North, A.C. (1999). The functions of music in everyday life: Redefining the social in music psychology. Psychology of Music, 27(1), 71.

Herrera, L., Cremades, R., y Lorenzo, O. (2010). Musical preferences in compulsory secondary education students: Influence of formal and informal education. Cultura y Educación 243; n, 22(1), 37-51.

Ibáñez, J. (1979). Interpretación y análisis. Más allá de la sociología. El Grupo de Discusión, técnica y crítica. (pp. 333-351). Madrid. Siglo XXI.

Korac, N. (1988). Functional, cognitive and semiotic factors in the development of audiovisual comprehension. Educational Communication \& Technology, 36, 6791

Kripedorff, K. (1990). Metodología de análisis de contenido. Teoría y práctica, Barcelona, Paidós.

Lévi-Strauss, C. (1969). Antropología Estructural. Bs As, Eudeba.

Ley Orgánica 2/2006) Título II, cap 1, artículo 71,76 y 77; RD 943/2003,18/06, BOE del 31; RD 1513/2006, de 7 de diciembre)

Lozano, J., Abril, G., \& Peña, C. (1982). Análisis del discurso: Hacia una semiótica de la interacción textual. Madrid. Cátedra.

McLaren, P., \& Kincheloe, J.L. (2008). Pedagogía crítica. Barcelona. Grao.

Medrano, C. \& Aierbe, A. (2008). Valores y contextos de desarrollo. Revista de Psicodidáctica, 13(1), 53-68. 
Molino, J. (1975). Fait musical et sémiologie de la musique. Musique En Jeu, 17, 37-62. París. Seuil.

Nattiez, J. (1990). Music and discourse: Toward a semiology of music Princeton University.

Novas, Iborra y Sampascual, (2007). Las metas académicas de los estudiantes de ESO en la clase de música. Revista de Psicodidáctica, 12 (1), 131-142.

Pérez, M. (1981): El universo de la música, Musicalis, Madrid.

Peirce, Charles S. (1938-1956). The Collected Papers, 8. Hartshorne, Weiss, and Burks (eds.). Cambridge: Harvard University.

Piaget, J. (1972). El nacimiento de la inteligencia. Madrid. Aguilar.

Porta, A. (1998). El metrónomo pulsional del rey León. Análisis de la banda sonora. Episteme. Valencia

Porta, A. (2007). Músicas públicas, escuchas privadas. Hacia una lectura de la música popular contemporánea. Aldea Global. Barcelona. UAB.

Porta, A. (2010). Qué escuchan los niños en la TV. UJI/Rivera. Castellón/Valencia

Porta, A. (2011). La oferta musical de la programación infantil de «TVE» como universo audible. Comunicar, 37, 177-185. (DOI: 10.3916/C37-2011-03-10).

Porta, A. \& Ferrández, R. (2009). Elaboración de un instrumento para conocer las características de la banda sonora de la programación infantil de televisión. RELIEVE, 15, 2. http://www.uv.es/RELIEVE/v15n2/RELIEVEv15n2_6. htm.

Pozo, J.I. (1989). Teorías cognitivas del aprendizaje, Madrid: Morata.

Prendergast, R. (1977). Film Music: a neglected art. New York: Norton and Company. Ramonet, I. (1998). La Aldea Global". Gedisa, Barcelona.

Reyes, J. (2006). Perpendicularidad entre arte sonoro y música en Cuadernos del Centro de Estudios en Diseño y Comunicación [Ensayos], 20. pp 9-21. Palermo. Universidad Palermo.

Schaeffer, P. (1966). Traté des Objets Musicaux. Paris. Seuil.

Schafer, M. (1977). The tuning of the world. Toronto, McClelland and Steward.

Sloboda, J. (2005). Exploring the musical mind: Cognition, emotion, ability, function. Oxford University.

Talens, J. (1995). Escritura contra simulacro. El lugar de la literatura en la era de la electrónica. Valencia. Eutopías.

Talens, J. \& Zunzunegui, S. (2007). Contracampo: Ensayos sobre teoría e historia del cine. Madrid. Cátedra.

Vygotsky, L.S. (1981). The instrumental method in psychology. The Concept of Activity in Soviet Psychology, 135-143. Cambridge, MA: MIT.

Westerkamp, H. (1994). De la Bauhaus al Paisaje Sonoro Instituto Goethe. Tokio.

Zamacois, J. (1987). Teoría de la Música 1 y 2. Barcelona.Labor.

Zunzunegui, S. (1989). Pensar la imagen. Madrid. Cátedra. 